\title{
Mujeres despojadas: representaciones de la violencia de género durante el Nacionalsocialismo en la obra Pálido criminal de Philip Kerr
}

\author{
Alicia ROMERo LÓPEZ \\ Departamento de Lengua Española y Teoría de la Literatura y Literatura Comparada \\ Universidad Complutense de Madrid \\ aliciafilol@gmail.com
}

\begin{abstract}
RESUMEN
La novela Pálido Criminal de Philip Kerr se desarrolla en el Berlín de 1938, cuando el estallido de la II Guerra Mundial es inminente. En este contexto se producen una serie de asesinatos de mujeres arias, de los que se hará cargo el detective Bernie Gunther, obligado a sumarse a las filas de la Kripo (Policía criminal) para intentar detener al asesino en serie antes de que siga matando. Esta obra rescata un tema relegado al olvido y llama la atención sobre el feminicidio, que pasó desapercibido en una contienda tan brutal como lo fue la II Guerra Mundial. La violencia contra la mujer ha sido, y aún hoy en día sigue siendo, un tema controvertido, que en este caso se trabaja a través de la novela negra, dotándola de un alcance social que no solo podemos ligar a nuestro pasado, sino también a nuestro presente.
\end{abstract}

Palabras clave: nacionalsocialismo, feminicidio, violencia de género, microviolencias, violación.

\section{Deprived Women: representations of gender violence during National Socialism in Pálido Criminal by Philip Kerr}

\begin{abstract}
Set in Berlin in 1938, Philip Kerr's The Pale Criminal takes place against the backdrop of the imminent Second World War. It is within this context that the detective Bernie Gunther sees himself embroiled in a series of fatal attacks on Aryan women, whose murders prompt him to join the Kripo (Criminal Investigation Agency) in order to find the serial killer before he strikes again. The novel brings to light a topic that has not received historical recognition and highlights the brutality of crimes against women within the already brutal context of the Second World War. Gender based violence was then, and continues to be, a controversial issue. In this instance the issue is approached by and through the criminal novel, providing a means through which we can uncover essential elements of the past that are just as relevant today.
\end{abstract}

Keywords: National Socialism, femicide, gender violence, microaggressions, rape. 


\section{Introducción}

Las noticias relacionadas con feminicidios que por desgracia tienden a ocupar día sí, día también las primeras planas de la prensa ${ }^{1}$ no nos deben llamar a engaño haciéndonos pensar que la violencia de género es una problemática meramente contemporánea, puesto que si concebimos el patriarcado como una estructura histórica podremos rastrear la evidencia de esta violencia en otros momentos de la historia. Las violencias de género van más allá de los asesinatos, punta visible del iceberg, son más profundas y afectan a la cotidianidad de nuestras vidas. Estas violencias invisibles, o microviolencias, serán estudiadas en la obra Pálido Criminal (1990) de Philip Kerr, ya que las mujeres que en ella se representan aparecen despojadas, en tanto que privadas de lo que gozan y tienen, y habiendo sido desposeídas de ello con violencia ${ }^{2}$.

Esta obra es la segunda parte de la trilogía Berlin Noir y está considerada una novela policíaca de corte histórico en la línea del hardboiled: «Philip Kerr’s Bernie Gunther series draws heavily on the American hardboiled detective genre: Gunther is a world-weary, wisecracking private investigator in the style of Raymond Chandler's Marlowe, transposed to the mean streets of Berlin during the Nazi regime» (Hall 2013: 293-294). El marco histórico, la Alemania nazi de 1938, es imprescindible para el análisis de la obra, ya que «the Holocaust is a constant presence in the novels, hovering just behind the stories, showing itself now and then through indirect references and allusions» (Lake 2016: 91). En concreto, la obra Pálido Criminal se basa en la investigación llevada a cabo por el detective Bernie Gunther, quien ha de volver a formar parte de la Kripo (Policía criminal) como Kriminalkommissar, para resolver el asesinato continuado de varias jóvenes arias. Este trabajo se centra en el análisis de las consecuencias y connotaciones que tienen estos asesinatos de género, ya que constituyen tan solo la continuación lógica y progresiva de un proceso de violencia patriarcal. En la obra Pálido Criminal se nos muestra la cara más violenta y machista del nazismo, durante el cual las mujeres jugaron un doble papel, ya que fueron politizadas y utilizadas por el régimen para dar una imagen patriótica, y por otro lado humilladas, reducidas a meros objetos reproductores. Como en tantos otros aspectos, constituye un error considerar el régimen nacionalsocialista como una anomalía, ya que en él la institución del patriarcado se encontraba tan presente y funcional como en cualquier otra sociedad no emancipada, entendiendo por patriarcado:

Patriarchy in its wider definition means the manifestation and institutionalization of male dominance over women and children in the family and the extension of male dominance over women in society in general. It implies that men hold power in all the important institutions of society and that women are deprived of access to such power. (Lener 1986: 239)

\footnotetext{
${ }^{1}$ Sirvan a modo de ejemplo los datos recogidos en el Boletín estadístico Mensual sobre Violencia de Género, publicado por el Ministerio de Sanidad, Servicios Sociales e Igualdad (http://observatorioviolencia.org/estadisticas/) que refieren el caso de 60 mujeres asesinadas solo en 2015.

2 Definición basada en la dada por el DRAE para el verbo despojar: «Privar a alguien de lo que goza y tiene, desposeerlo de ello con violencia».
} 
Por lo tanto, el análisis socioliterario aquí llevado a cabo está asentado sobre la crítica de la estructura patriarcal. La literatura nos sirve, en este caso, como reflejo de la historia y de los diversos problemas de la mujer alemana, tema este que muchas veces ha quedado relegado a un segundo plano por la magnitud de la barbarie genocida y exterminadora del régimen nacionalsocialista.

\section{La mujer durante el Nacionalsocialismo}

La cuestión de género fue uno de los elementos claves de la ideología nacionalsocialista, ya que uno de sus pilares, la higiene racial ${ }^{3}$, recaía, en gran parte, en la maternidad y por ende en la mujer. Desde 1933 las políticas a este respecto fueron in crescendo y las libertades alcanzadas por las mujeres durante la República de Weimar (1918-1933) desapareciendo. Al tener que ser madre y esposa, la mujer fue poco a poco recluida a la esfera privada, al hogar, negándosele espacios políticos y educativos:

Desde los años veinte las mujeres trabajaban en todos los sectores públicos y privados y enarbolaban los ideales emancipatorios sobre los principios del sufragismo femenino, la educación y el trabajo. [...] Las mujeres alemanas no solo reivindicaban un espacio propio en el ámbito público sino que además irrumpían con fuerza en ámbitos tradicionalmente masculinos. Las mujeres ocupaban puestos académicos en las universidades, irrumpían con fuerza en el ámbito político [...] (Beteta Martín 2012: 111-112)

Con el ascenso de Hitler al poder se expulsó a las mujeres de todo espacio académico e intelectual, vetándoles la entrada, por ejemplo, a la docencia universita-

${ }^{3}$ El concepto de higiene racial se usa como sinónimo de eugenesia, cuya definición según el DRAE es: «Estudio y aplicación de las leyes biológicas de la herencia orientados al perfeccionamiento de la especie humana». Los estudios relativos a este campo comenzaron con anterioridad a la llegada de Hitler al poder, aunque fue durante el III Reich cuando las políticas basadas en estas ideas fueron puestas en práctica con más vehemencia. Sin embargo, desde finales del s. XIX genetistas alemanes centraron sus estudios en cómo mejorar la raza a través de la genética. En 1905 Alfred Ploetz funda la Gesellschaft fur Rassenhygiene (Sociedad para la Higiene Racial). Durante los años de la República de Weimar estos trabajos se siguieron desarrollando: «Thus throughout the Weimar years, as during the Empire, the movement was concerned first and foremost with boosting Germany's national efficiency and cultural productivity» (Faith Weiss 1978: 225). El problema radica en que estos estudios se movían en un terreno ideológico complejo, en el que el racismo (en favor de la raza aria) jugaba un papel importante: «Like the great majority of educated whites in Europe and North America of their day, all race hygienists accepted the racial and cultural superiority of Caucasians as a matter of course (Faith Weiss 1978: 194). Uno de los pasos que anticipó lo que ocurriría posteriormente fue la promulgación de una ley en 1932 por la que se legalizaba la esterilización voluntaria de determinados individuos con trastornos genéticos (cf. Faith Weiss 1978: 225). Durante el nacionalsocialismo las políticas de higiene racial se convirtieron en una forma de perpetuar la raza aria y su máxima expresión fue la implantación de la ley Gesetz zur Verhutung erbkranken Nachwuchses (Ley para la prevención de descendencia con enfermedades hereditarias), con la que la esterilización pasó de voluntaria a obligatoria (cf. Faith Weiss 1978: 229). 
ria. Con esta exclusión del mundo laboral lo que se quería conseguir era que estas se dedicaran por completo al cuidado de la casa y de los hijos. En un discurso público Goebbels afirmó: «Tener niños y educarlos es una gran tarea. Mi madre es la mujer a la que más respeto, y está alejadísima del intelecto, y tanto más próxima a la vida» (Beteta Martín 2012: 111). En 1933 se creó el Deutsches Frauenwerk (Servicio femenino del trabajo), cuya misión era preparar a las jóvenes alemanas para ser buenas madres y amas de casa. Al comienzo, la pertenencia a esta organización fue voluntaria, pero a partir de 1936 pasó a ser obligatoria, al menos durante seis meses, y comenzó a denominarse Reichsarbeitsdients der weiblichen Jugend (Servicio de trabajo para la juventud femenina). Esta organización ayudó a paliar el paro, pues las mujeres desempleadas se las colocaba en algún puesto no remunerado para ayudar a familias con muchos hijos, cuidar de enfermos, para realizar labores del campo e incluso las mandaban a zonas recientemente colonizadas para que ayudaran a las familias en tareas domésticas (Rodríguez Luján 2014: 33-34). Con ello, conseguían que las jóvenes alemanas difundieran y consolidaran la ideología nacionalsocialista.

La propaganda nacionalsocialista quiso erigir a la mujer como el símbolo de la madre patria, que había de continuar con la reproducción de la raza aria, elemento indispensable para la supremacía y bienestar del pueblo alemán. Sin embargo, esta idea de la maternidad representa dos caras de una misma moneda, ya que por un lado se dio la explotación sexual de las mujeres arias, y por otro, la creación de determinadas leyes eugenésicas para que las mujeres «racialmente inferiores» no tuvieran hijos.

Centrándonos en el primer aspecto, el de la explotación sexual, es necesario llamar la atención sobre la creación de Lebensborn, una organización fundada por Heinrich Himmler para reproducir la raza aria. Creada en 1935 en Alemania, posteriormente tuvo sedes en diferentes países como Noruega, Austria, Holanda, Bélgica o Dinamarca. Esta asociación Lebensborn fue constituida para que los alemanes considerados arios, después de ser sometidos a pruebas médicas que verificaran los estándares de pureza, se reprodujeran y los recién nacidos fueran adoptados por familias pertenecientes a las SS (Whittock 2011: 154). Muchas mujeres formaron parte de este proyecto, en el que incluso se permitió tener hijos en secreto a las que no estaban casadas, siempre que cumplieran con el estereotipo ario. Más allá de su componente racista, este proyecto comportaba la cosificación de las mujeres en tanto meras máquinas de producción de hijos.

Por otro lado, la higiene racial conllevó la creación de determinadas leyes eugenésiscas que impidieran la reproducción de los «no racialmente puros». En 1933 se creó la Ley para la prevención de descendencia con enfermedades hereditarias (Gesetz zur Verhütung erbranken Nachwuchses), que fue implementada en 1934 y que permitió la esterilización de miles de ciudadanos que pudieran tener trastornos genéticos, los cuales también integraban defectos emocionales o intelectuales tales como supuesta debilidad mental, esquizofrenia, epilepsia y desórdenes maniacodepresivos (Bock 1994: 197). Esta política nacionalsocialista de esterilización fue un paso previo al programa eugenésico Aktion T4 o «prevención de la vida sin valor», que comenzó en 1939 y asesinó a más de 200000 personas (Bock 1994: 200). La estructura que creó el nacionalsocialismo en torno al concepto de mujer no hizo más 
que reafirmar la ideología patriarcal y subyugar a la mujer, convirtiéndola en un mero objeto reproductor del que hacer uso.

\section{Microviolencias: las violencias invisibles}

El sociólogo Johan Galtung clasificó los diferentes tipos de violencias en tres grandes bloques: estructural, cultural y directa, siendo las dos primeras parte de las violencias invisibles y la última la visible. En este texto trabajaremos con ellas aplicándolas a la problemática de género, pues la estructura patriarcal es básica para la reproducción y legitimación de la violencia machista. A este respecto, se debe señalar que el patriarcado aparece en el texto de Galtung como una ejemplificación de la violencia estructural: "Patriarchy is then seen as an institutionalization of male dominance in vertical structures, with very high correlations between position and gender, legitimized by the culture (e.g., in religion and language), and often emerging as direct violence with males as subjects and females as objects» (Galtung 1996: 40). El sistema patriarcal como violencia invisible está reforzado por una serie de microviolencias, las cuales se definen como toda forma de violencia contra la mujer que está de tal forma asimilada en la sociedad que se convierte en invisible. Esta idea está en consonancia con la definición de (micro)Machismo que aparece en el texto Micromachismos, la violencia invisible en la pareja (1998) de Luis Bonino:

Los $\mathrm{mM}$ son prácticas de dominación y violencia masculina en la vida cotidiana, del orden de lo «micro», al decir Foucault de lo capilar, lo casi imperceptible, lo que está en los límites de la evidencia. [...] Los mM son microabusos y microviolencias que procuran que el varón mantenga su propia posición de género creando una red que sutilmente atrape a la mujer, atentando contra su autonomía personal [...]. (4)

Este trabajo analiza cuál fue la consideración de la mujer alemana durante el nacionalsocialismo centrándonos en cuatro microviolencias que se reflejan en la obra Pálido Criminal: la usurpación de la maternidad, la violencia estética, el miedo como forma de violencia y la cosificación de la mujer; para así, posteriormente, observar cómo estas violencias invisibles se convierten en visibles, representadas en este caso como feminicidios.

\subsection{Usurpación de la maternidad}

La maternidad ha servido a lo largo de los siglos como un elemento de control patriarcal, es decir, a la mujer se le ha usurpado su derecho a la maternidad como una forma de reclusión a la esfera privada. Consideramos que la usurpación ha venido dada por la prohibición no explícita a las mujeres a poder decidir si querían ser madres o no y cómo querían serlo. Al negarles la maternidad se convirtió a las mujeres solteras en solteronas, con toda la carga despectiva que la palabra conlleva, y a las no fértiles en sujetos no válidos y excluidos del sistema patriarcal. En el caso 
concreto del nacionalsocialismo no solo hemos de hablar de usurpación, ya que las mujeres estaban prácticamente obligadas a ser madres, sino de cosificación de la maternidad, en tanto que dejó de tener un sentido natural para pasar a ser industrial. A la mujer se la convirtió en una fábrica de hijos que asentaran y mantuvieran las características arias del pueblo alemán:

-En la nueva Alemania -dijo [Heydrich]-, de lo que se trata es de detener el deterioro de la familia, ¿saben?, y establecer una comunidad de sangre en la nación. Las cosas están cambiando. Por ejemplo, ahora solo hay 22.787 vagabundos en Alemania, 5.500 menos que al principio del año. Hay más matrimonios, más nacimientos y la mitad de divorcios. Podrían preguntarme por qué la familia es tan importante para el partido. Se lo diré. Los niños. Cuanto mejores sean nuestros hijos, mejor será el futuro de Alemania. (Kerr 2010: 274)

La novela de Kerr pone de relieve cómo el nacionalsocialismo, al querer renovar la raza y crear sujetos «perfectos», mitificó la figura de la madre. Además, y como se verá en el siguiente apartado, la violencia estética fue una de las claves para someter a la mujer, pues tan solo las que cumplían con el estereotipo ario eran dignas de tener hijos que construyeran la nueva patria alemana: «Con objeto de mejorar la 'calidad' de la población, la 'regeneración racial' o la edificación racial (Aufartung), el Estado nacionalsocialista llevó a cabo una política destinada a evitar nacimientos o antinatalista, a través de la esterilización masiva obligatoria» (Bock 1996: 402). Por lo tanto, nos encontramos con dos caras de la misma moneda, por un lado la mitificación de la maternidad, como elemento salvador de la raza, y por otro, las políticas antinatalistas. Ambas caras son una forma de sometimiento de la mujer a la ideología dominante, si bien, la última fue desde la esterilización al asesinato de miles de mujeres judías o «no arias».

La novela de Kerr refleja la idea del culto a la maternidad a través de los asesinatos de jóvenes arias, es decir, las futuras madres: «[...] son las jóvenes madres del futuro a quienes está matando. Máquinas productoras de niños que serán nuevos miembros del partido» (Kerr 2010: 289). En esta obra se observa, por tanto, cómo la maternidad, e incluso la figura de las mujeres alemanas, fue usada como un elemento político, privando de la libertad a decidir a miles de mujeres.

\subsection{Violencia estética}

[...] la violencia estética consiste en la promoción por parte de los medios de comunicación y difusión masiva, la industria de la moda, de la música y el mercado cosmético, de unos cuerpos «perfectos», los cuales no son más que cuerpos ficticios, irreales, concebidos como ideal, como deber ser, como patrón a seguir, y donde las particularidades físicas de las mujeres son denominadas «imperfecciones», que de acuerdo a los criterios de belleza reproducidos y transmitidos necesariamente han de ser intervenidas y suprimidas, o en el menor de los casos corregidas. (Pineda 2013)

En el caso del nazismo este tipo de violencia está muy ligada al ideal de belleza ario, ideal que sustenta, como se ha visto, gran parte de su ideología, y por el cual 
comienzan a crearse leyes para la higiene racial. En la obra de Kerr, todas las chicas asesinadas «responden al estereotipo ario» (Kerr 2010: 285), por lo que el asesino está escogiendo cuidadosamente a sus víctimas entre aquellas muchachas que cumplen con esas características. El texto, sin embargo, no deja de estar cargado de ironía, pues una joven, Sara Hirsch, salva su vida por el hecho de tener un apellido judío, ya que, sin embargo, las características físicas de la misma son arias: «rubia de ojos azules» (Kerr 2010: 355) «habló con el chofer y le preguntó si yo no era un hermoso ejemplo de las doncellas alemanas» (Kerr 2010: 356). Con esto, el autor deja ver las incongruencias de una ideología carente de sentido y que basaba sus ideales estéticos en las palabras escritas por Tácito en De las Costumbres, Sitios y Pueblos de la Germania:

Estoy casi convencido de que los germanos son indígenas y que de ningún modo están mezclados con otros pueblos, bien como resultado de emigraciones, bien por pactos de hospitalidad. [...] Me adhiero a la opinión de que los pueblos de Germania, al no estar degenerados por matrimonios con ninguna de las otras naciones, han logrado mantener una raza peculiar, pura y semejante solo a sí misma. De aquí su constitución física, en lo que es posible en un grupo tan numeroso, sea la misma para todos: ojos fieros azules, cabellos rubios, cuerpos grandes y capaces solo para el esfuerzo momentáneo [...] (Tácito 1981: 115-116)

El hecho de que las mujeres tuvieran que presentar unos rasgos tan concretos hizo que algunas de ellas, incluso ya siendo rubias, usaran tintes para aclarar aún más su cabello: tinte para el pelo Schwarzkopf Extra Blonde «seis de cada diez chicas lo usan» (Kerr 2010: 282). Este tipo de violencia estética, que a veces queda enmascarada tras una fachada ilusoria de moda y belleza, realmente constituye una forma de subyugación de la mujer a una serie de cánones impuestos por el poder, en este caso el gobierno nacionalsocialista. En esta época, además, la mujer no debía maquillarse, pues esto era un símbolo de perversión, más asociado a las prostitutas. Estas ideas fueron inculcadas a las jóvenes no solo a través del Servicio de trabajo para la juventud femenina, sino que ya en 1930 se había creado la Bund Deutscher Mädel (Asociación de Muchachas Alemanas), como rama femenina de las Juventudes Hitlerianas, y en 1936 el ingreso se tornó obligatorio para las jóvenes entre 10 y 18 años. En la novela de Kerr se explicita que algunas de las jóvenes formaban parte de la BdM: «Mi hija [Irma Hanke] era una chica de la BdM, elogiada en su clase de teoría racial como ejemplo perfecto del tipo ario» (Kerr 2010: 334). Posteriormente, en 1938 se creó la sección Glaube und Schönheit (Fe y Belleza) para chicas de entre 17 y 18 años (Rodríguez Luján 2014: 20). Las directrices que se impartían a estas jóvenes iban encaminadas no solo a cómo comportarse (qué vestir, nociones de moda, etc.), sino que se las preparaba para el matrimonio. Por todo ello, también consideramos que dentro de la violencia estética es posible incluir la creación e imposición de cánones (sociales, de comportamiento, etc.) preconcebidos que coartan las libertades de las mujeres. La novela de Kerr refleja no solo el sometimiento de las mujeres a las doctrinas nacionalsocialistas, sino que hace una crítica de misma, por ejemplo a través de cómo estas rebelaban ante el sistema fumando, algo mal visto en la mujer: 
-Creía que vosotras, las chicas de la Fe y la Belleza, no fumabais -dije tratando de ocultar mi irritación.

- ¿Seguro? - dijo con una sonrisa de suficiencia-. Hay muchas cosas que no nos animan a hacer. Se supone que no debemos hacer esto, se supone que no debemos hacer lo otro. Casi todo parece ser pecaminoso en esta época, ¿no? Pero lo que yo digo siempre es: si no puedes hacer todas esas cosas malas cuando todavía eres joven para disfrutarlas, entonces, ¿qué sentido tiene hacerlas? (Kerr 2010: 292)

Esta cita nos sirve como forma de crítica a un sistema que consideraba que lo «pecaminoso» debía ser extirpado de la sociedad, más aún del comportamiento de las mujeres. Debemos asociar la represión de la mujer como sujeto social al sistema patriarcal, del cual se valió el nacionalsocialismo para sustentar parte de su concepción ideológica.

\subsection{Miedo como forma de violencia}

La obra de Kerr es doblemente interesante, ya que por un lado muestra la situación de la mujer alemana durante el nacionalsocialismo, pero por otro, trae a la actualidad determinadas problemáticas sociales asociadas a la mujer que aún hoy en día siguen vigentes. En este sentido, se ha de señalar el miedo de las mujeres a ser agredidas, es decir, el miedo como forma de violencia: «La percepción del miedo a ser agredida es tan importante o más que la posibilidad real estadística de serlo, porque es el miedo el que impide a las mujeres salir de casa y moverse por el espacio urbano» (Sánchez de Madariaga 2004).

La alarma continua y el miedo que se infunde en las mujeres desde que son pequeñas, se refleja en la obra de Kerr a través de las situaciones en las que los agresores raptan a sus víctimas, y en cómo reaccionan las autoridades ante esta amenaza: creando una campaña publicitaria «para que las chicas fueran más conscientes de la necesidad de tener cuidado» (Kerr 2010: 287). Las circunstancias en las que las jóvenes desaparecen son cotidianas: Brigitte Hartmann iba a visitar a su tía en Reinickendorf, Christiane Schulz desaparece después de salir de los estudios de radio donde cantaba, Zarah Lischka estaba de compras, Lotte Wintter salía de casa para ir a equitación, Irma Hanke al ir a hacer una colecta o Liza Ganz que desaparece mientras iba a hacer la compra al mercado del barrio. La inseguridad que las mujeres experimentan en situaciones puramente cotidianas es atribuible a un sistema patriarcal que desde hace siglos mantiene una política del miedo, construyendo así socialmente a las mujeres como sujetos frágiles expuestos permanentemente a la violencia del hombre. El hecho de que todas las mujeres sean consideradas potenciales víctimas, idea que se fija desde cualquiera ámbito comunicativo (familia, medios de comunicación, campañas, etc.), las priva de determinadas libertades:

El miedo a una agresión sexual parece, por tanto, un guión fijo transmitido a todas las chicas jóvenes que, consciente o inconscientemente, emplean mecanismos de protección frente a este miedo pre-programado: coger un taxi para volver a casa, volver en compañía, hacer una llamada cuando llegan a casa para confirmar a alguien que están 
bien, pasar por ciertos lugares corriendo... Se trata de mecanismos que las jóvenes emplean continuamente, de una forma normalizada y naturalizada, pero que suponen una limitación importante de su libertad. (VV.AA. 2011: 68)

La novela de Kerr no hace más que poner de relieve una problemática social sufrida por las mujeres desde hace siglos. El recorte de las libertades de las mujeres a través del miedo a ser agredidas sexualmente se plasma en esta novela como un suceso realista, que acaba, además, en asesinato.

\subsection{Cosificación de la mujer}

El protagonista, Bernie Gunther, constantemente actúa como opositor al régimen nacionalsocialista, sin embargo, en cuanto a la forma de tratar a las mujeres es contradictorio. Por un lado, parece respetarlas y afirma que la mujer sirve para mucho más que ser un mero objeto reproductor:

Señores, me gustaría presentarles a Frau Kalau vom Hofe. He dicho Frau porque no se le permite utilizar el título de doctora, aunque lo es, porque es muy evidente que es una mujer y todos sabemos, ¿no es así?, que el lugar de una mujer está en el hogar, produciendo reclutas para el partido y preparando la cena de su marido. En realidad es psicoterapeuta y una reconocida experta en ese pequeño e insondable misterio al que nos referimos como la «mente criminal». (Kerr 2010: 285).

Esta forma de cosificación de la mujer, relegándola de nuevo a la esfera privada y expulsándola de la pública, espacio reservado para los hombres, fue frecuente no solo en el nacionalsocialismo, sino también durante otros regímenes dictatoriales como pudo ser el franquismo. Esta idea contrasta con la explicitada en líneas anteriores, de mitificación de la mujer y de la maternidad. Así, queda claramente reflejado, que a la mujer no se la alabó en tanto que mujer, sino como mero objeto de reproducción.

Sin embargo, debemos señalar, que sí existieron determinadas mujeres que formaron parte muy activa de la «cruzada nacionalsocialista», y que trabajaron como SS Oberaufseherin (guardia femenina) en campos de exterminio. Algunos de los nombres más destacados son Irma Grese, Ilde Koch, Dorothea Binz o Hermine Braunsteiner. Por lo tanto, es importante que en este trabajo quede reflejado que la figura de la mujer alemana no solo fue víctima, sino también agresora.

Por otro lado, y volviendo a la serie policíaca Berlin Noir, se ha de destacar que aunque la actitud de Bernie Gunther frente a la doctora Frau Kalau vom Hofe en algunos momentos es de estima y halago, en otros casos la percibe como un mero objeto sexual: «Sonreí, apreciando su tranquila resistencia, así como los hermosos pechos que tensaban la tela de su blusa» (Kerr 2010: 288). En este punto, es importante señalar que las mujeres judías también son tratadas de la misma forma, pues la violencia sexual no entiende de razas:

[...] frente a la desindividualización y desexualización derivada del trato que reciben por parte de las autoridades de las SS que mutila la subjetividad y el cuerpo de las 
mujeres, se mantienen actitudes que las sexualizan y les hace conscientes de su categorización como objetos sexuales. Las leyes de Nüremberg prohibían las relaciones sexuales entre los alemanes y las mujeres judías sin embargo, aunque esta restricción se mantuvo entre los altos mandos de las SS que dirigían los campos de concentración exterminio, se registran casos de abusos sexuales y violaciones por parte sobretodo de los mandos intermedios de las SS y de prisioneros de alto rango. (Beteta 2012: 124)

Este hecho se pincela en la obra de Kerr al hablar de una prostituta de raza judía: «Hay una putilla judía en ese masajeadero a donde vamos - dijo con entusiasmo-. Una auténtica bomba. Tiene una almeja que es como el interior de un congrio, como un largo músculo succionador; de la clase que puede tragarte como si fueras una sardinilla y lanzarte afuera por el culo» (Kerr 2010: 304). Las mujeres «no arias» sufrieron, por tanto, una doble explotación: la de no pertenecer a una determinada raza y por otro, la cosificación sexual de sus cuerpos.

\section{Violencia(s) Visible(s): Asesinatos y violaciones de mujeres en Pálido Criminal}

Por último, en el análisis de la obra Pálido Criminal es fundamental trabajar con las violencias visibles, que en este caso concluyen con el asesinato de siete jóvenes arias entre los quince y los diecisiete años: Brigitte Hartmann, Zarah Lischka, Lotte Wintter, Irma Hanke, Emmeline Steininger, Liza Ganz y Christiane Schulz. Sin embargo, y como ya se ha mencionado, estas chicas ya habían sido violentadas anteriormente. Las siete jóvenes desaparecen durante sus actividades rutinarias y aparecen muertas días después. Estos asesinatos de jóvenes arias son atribuidos en un primer momento a un ciudadano judío, sin embargo, mientras este está en el calabozo se descubre un nuevo cuerpo. Por ello, la Kripo recurre a Bernie Gunther para que les ayude a encontrar al asesino en serie. Este detective, atípico para la época, pues es contrario al régimen nacionalsocialista, será el que a través de sus percepciones nos guíe por los entresijos policiales más oscuros del nazismo. Los asesinatos de las jóvenes no paran de sucederse y algunas de las escenas descritas en la obra son brutalmente violentas: «La vagina no estaba dañada y no había sido penetrada -continuó Illmann-. Por el contrario, el ano estaba muy distendido, permitiendo introducir los dedos. Las pruebas en busca de espermatozoides fueron positivas» (Kerr 2010: 283). Las muchachas arias no solamente son asesinadas, sino que debido a la cosificación sexual, anteriormente señalada, también son violadas y agredidas sexualmente: «No obstante, la entrada vaginal mostraba huellas de arañazos y había huellas de magulladuras en la pelvis, indicando que había sido penetrada... y con violencia» (Kerr 2010: 284). Los agresores se deleitan en la brutalidad de su acto: «Se la tiró y la desgarró algo al hacerlo. Bueno, eso sería de esperar. La chica era virgen, diría yo. Incluso le dejó huellas de uñas en la membrana mucosa» (Kerr 2010: 332). Kerr al describir tan detalladamente este tipo de escenas coloca al lector en una posición desagradable, pero que consideramos necesaria en tanto que al exponernos de manera brutal a las violaciones nos vemos en la obligación de reflexionar sobre ellas. Esta interpretación crítica es necesaria para entender no solo 
cómo la mujer era tratada hace casi un siglo, sino para poder ver reflejadas determinadas actitudes en nuestra actualidad, sirviéndonos la literatura como nexo para reflexionar sobre nuestro presente a través de nuestro pasado.

La obra de Kerr, sin embargo, no solo pone sobre el tablero las atrocidades de la violencia de género, sino que las introduce como parte de una trama política en la que varias personalidades importantes del régimen nazi (Otto Rahn, Karl Maria Wiligut o también llamado Weisthor, e incluso Himmler) se encuentran tras los asesinatos. Esta vertiente política de los asesinatos pretende acusar a los judíos del ataque a las jóvenes arias, para así tener un motivo concreto para cargar contra ellos. La novela de Kerr se salda con la resolución del caso por parte de Bernie Gunther, sin embargo, las últimas líneas están dedicadas a la Kristallnacht. Con ello Kerr nos muestra los largos tentáculos del nacionalsocialismo que asesinó, torturó y humilló a miles de personas por no responder a unos ideales estéticos o políticos, y que en el caso de las mujeres fueron doblemente subyugadores.

\section{Conclusiones}

Como se ha podido comprobar a través de este breve estudio, la violencia de género fue una de las problemáticas sociales más importantes durante el nacionalsocialismo, aunque en muchos casos quedara relegada a un segundo plano. La cosificación y politización tanto de la mujer como de la maternidad constituyeron una de las bases en las que se sustentó la ideología nazi. Por lo tanto, la mujer sirvió como elemento político, a través del cual perpetuar la raza aria y exterminar cualquier otra. Sin embargo, esta exaltación y mitificación de la maternidad no hizo que la mujer tuviera un papel relevante socialmente, sino que se la siguió considerando un mero objeto sexual. En este trabajo nos hemos centrado en el papel de la mujer alemana durante los primeros años del nacionalsocialismo, pero no debemos dejar de mencionar a todas aquellas mujeres judías, gitanas, comunistas, etc. que sufrieron una doble explotación: como mujeres y como «no arias». La mujer, en muchos casos, fue utilizada a placer del hombre y de las autoridades, relegándola a la esfera privada. La obra Pálido Criminal de Philip Kerr no solo nos hace reflexionar sobre la situación de las mujeres durante en nacionalsocialismo, sino que refleja una problemática social, como es la violencia de género, que hoy en día sigue constituyendo una de las problemáticas sociales más importantes.

\section{Referencias bibliográficas}

Beteta MartíN, Yolanda (2012): «La feminidad normativa y la violencia sexual en el III Reich. La deconstrucción de las identidades femeninas y la explotación sexual de las mujeres en los campos de concentración y exterminio». El Futuro del Pasado: revista electrónica de historia 3: 107-135.

BocK, Gisela (1994): «Antinatalism, maternity and paternity in National Socialist racismo», en Nazism and German Society, 1933-1945, London and New York: Routledge, pp. 110-140. 
BONINO, Luis (1998): Micromachismos, la violencia invisible en la pareja. Disponible en: http://www.luisbonino.com/pdf/mM96.pdf

FAITH WEISS, Sheila (1978): «The Race Hygiene Movement in Germany». Osiris 3: 193236.

Galtung, Johan (1996): Peace By Peaceful Means: Peace and Conflict, Development and Civilization. London: SAGE.

HALL, Katharina (2013): «The 'Nazi Detective' as Provider of Justice in Post-1990 British and German Crime Fiction: Philip Kerr's The Pale Criminal, Robert Harris's Fatherland, and Richard Birkefeld and Göran Hachmeister's Wer übrig bleibt, hat recht». Comparative Literature Studies 50 (2): 288-313.

KERR, Philip (2010): Trilogía Berlinesa. Barcelona: RBA.

LAKE, Anthony (2016): «'But What's One More Murder?' Confronting the Holocaust in Philip Kerr's Bernie Gunther Novels». ATLANTIS. Journal of the Spanish Association of Anglo-American Studies 38 (1): 89-107.

LERNER, Gerda (1986): The Creation of Patriarchy. New York: Oxford University Press.

PINEDA, Esther (2013): «La violencia estética contra la mujer». Palabra de mujer. Disponible en: https://palabrademujer.wordpress.com/tag/violencia-estetica-contra-la-mujer/

Rodríguez LujÁn, David (2014): Las Mujeres, Alemania y el Nazismo. [n/a]: Lulu.

SÁNCHEZ DE MADARIAGA, Inés (2004): Urbanismo con perspectiva de género. Instituto Andaluz de la Mujer. Disponible en: http://www.generourban.org/documentos/98.pdf

TÁCITO, Cayo Cornelio (1981): Vida de Julio Agrícola, Germania, Diálogo de los oradores. Madrid: Editorial Gredos.

VARELA, Nuria (2016): Feminismo para principiantes. Barcelona: Ediciones B.

VV.AA. (2011): Agresiones sexuales. Cómo se viven, cómo se entienden y cómo se atienden. Servicio central de publicaciones del Gobierno Vasco. Disponible en: http:/www.emakunde.euskadi.eus/contenidos/informacion/vcm_guias_publicac_folletos/es_def/adjuntos/pub.agresiones.sexuales.como.viven.cas.pdf

WhitTock, Martyn (2011): A Brief History of The Third Reich: The Rise and Fall of the Nazis. Londres: Robinson. 